\title{
Estudios en las Apocynaceae Neotropicales VI: Una nueva especie de Prestonia (Apocynaceae, Apocynoideae) para Brasil.
}

\author{
J. Francisco MORALES
}

\section{RESUMO}

Uma nova espécie de Prestonia R. Br., endêmica dos estados do Acre e Amazonas, Brasil, é descrita e ilustrada e se discute as relações com taxas relacionadas.

\section{PALAVRAS CHAVE}

Apocynaceae, Apocynoideae, Echiteae, Prestonia, Brasil.

\section{Studies in Neotropical Apocynaceae VI: A new species of Prestonia (Apocynaceae, Apocynoideae) from Brazil.}

\author{
ABSTRACT \\ A new species of Prestonia R. Br., endemic to the states of Acre and Amazonas, Brazil, is described and illustrated. In \\ addition, the relationships with related taxas are discussed.
}

KEY WORDS

Apocynaceae, Apocynoideae, Echiteae, Prestonia, Brasil.

La familia Apocynaceae es particularmente abundante en Brasil, con cerca de 376 especies y 41 géneros reportados (Sales, 1993), donde varios géneros (e.g., Aspidosperma, Himatanthus, Laxoplumeria, Mandevilla) tienen secciones cuyos miembros estan mayormente confinados a esta región geográfica. El género Prestonia, ampliamente distribuido desde México a Argentina y las Islas del Caribe, tiene su centro geográfico de endemismo en Brasil, donde cerca de 7 especies endémicas (P. amazonica (Benth. ex Müll. Arg.) J. F. Macbr., P. babiensis Müll. Arg., P. denticulata (Vell.) Woodson, $P$. didyma (Vell.) Woodson [incluyendo $P$. perplexa Woodson], P. dusenii (Malme) Woodson, $P$. macroneura (Müll. Arg.) Woodson y P. solanifolia (Müll. Arg.) Woodson) son conocidas (Woodson, 1936).

La última monografía del género fue hecha por Woodson (1936), quién reconoció 61 especies. En los últimos años, revisiones parciales para algunas secciones en áreas geográficas definidas han sido hechas (Morales, 1997), pero en general, la taxonomía del género se ha mantenido confusa. Durante la realización de una monografía de Prestonia para la serie Flora Neotrópica, un total de 55 especies han sido reconocidas, de las cuales 23 ocurren en
Brasil (Morales, en prep.). Una nueva especie, perteneciente a la sección Tomentosae (Woodson, 1936) y restringida a los estados de Acre y Amazonas fue encontrada. Por lo tanto, es descrita a continuación.

Prestonia acrensis J. F. Morales, sp. nov. Tipo. Brasil. Acre: Cruzeiro do Sul, base del proyecto RADAM/Brasil, 3 Mar 1976 (fl), Ramos \& Mota 249 (holotipo, INPA, fotocopia en INB) (Figura 1).

Frutex scandens, ramulis hirtellis. Folia elliptica, 10-12 x 7-8.5 cm, hirtella, apice acuminata, basi rotundata vel subcordata, petiolis $0.4-0.6 \mathrm{~cm}$ longis. Inflorescentia racemosa, lateralis, bracteis $3-13 \mathrm{~mm}$ longis, foliaceis; calycis laciniae anguste ellipticae, $10-13 \mathrm{~mm}$ longae, hirtellae; corola hypocrateriformis, tubo extus hirtello, 9-14 mm longo, corona lobis $2 \mathrm{~mm}$, paulo exsertis; antherae 4.5-5 $\mathrm{mm}$. Folliculi ignoti.

Liana, ramitas moderada a esparcidamente hirsutas, secreción desconocida, coleters interpeciolares conspicuos, 2-2.5 mm de largo. Hojas: lámina 10-12 x 7-8.5 cm, elíptica, acuminada apicalmente, redondeada a levemente cordada basalmente, membranácea, no revoluta marginalmente, 


\section{ACTA \\ AMAZONICA}

ESTUDIOS EN LAS APOCYNACEAE NEOTROPICALES VI: UNA NUEVA ESPECIE DE Prestonia (APOCYNACEAE, APOCYNOIDEAE) PARA BRASIL moderadamente hirsuta adaxialmente, densa a moderadamente hirsuta abaxialmente, indumento amarillo, venación secundaria y terciaria impresa, pecíolo $0.4-0.6 \mathrm{~cm}$. Inflorescencia racemosa, axilar, más corta que las hojas adyacentes, densamente aglomerada, con muchas flores, densa a moderadamente hirsuta; pedúnculo menos de 1.1 cm de largo, algo leñoso; pedicelos 0.3-0.6 cm; brácteas 0.3-1.3 cm, foliáceas a subfoliáceas; base del cáliz corta e inconspicua, no campanulada; sépalos 10-13 x 3-4 mm, libres a lo largo de su entera longitud, membranáceos, angostamente elípticos, cortamente acuminados, no reflexos apicalmente, densamente hirsutos, foliáceos, coleters ca. 2 $\mathrm{mm}$ de largo, enteros; corola hipocrateriforme, amarilla a verde amarillenta, densamente hirsuta exteriormente, tubo 9-14 x $3 \mathrm{~mm}$, recto, lóbulos coronales ca. $2 \mathrm{~mm}$, apicalmente exsertos, corona anular algo inconspicua, ca. $1 \mathrm{~mm}$, lóbulos

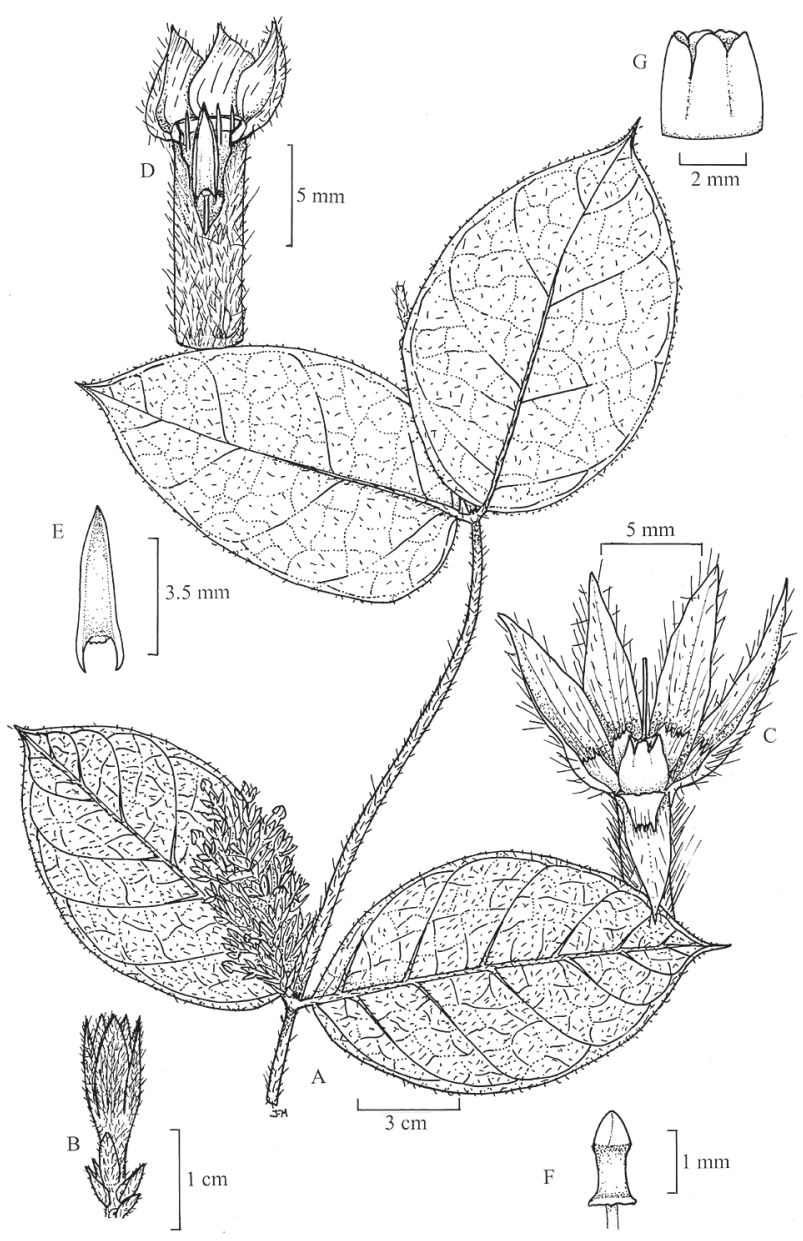

Figura 1 - Prestonia acrensis (Ramos \& Mota 249, INPA). A. Ramita con inflorescencia. B. Cáliz, pedicelos, y brácteas florales. C. Cáliz con los sépalos separados, mostrando los coleters y el nectario pentalobulado. D. Tubo de la corola parcialmente abierto, mostrando la corona anular, lóbulos coronales y anteras apicalmente exsertas. E. Antera, vista dorsal. F. Cabeza estigmática. G. Detalle del nectario.
4-5 x 2-3 mm, ovados; anteras 4.5-5 mm, muy diminuta e inconspicuamente puberulentas a glabradas dorsalmente, apicalmente exsertas; ovario ca. $1 \mathrm{~mm}$ de largo, densamente hirsuto, cabeza estigmática ca. $1.5 \mathrm{~mm}$, disco $2-3 \mathrm{~mm}$, levemente penta-lobulado. Folículos desconocidos.

Distribución. Esta especie es conocida solamente de los estados de Amazonas y Acre, Brasil, donde crece en bosques muy húmedos, bajo elevaciones de $200 \mathrm{~m}$. Especímenes con flores fueron recolectados en Marzo y Mayo.

Prestonia acrensis está cercanamente relacionada con P. calycina Müll. Arg. y P. ipomaeifolia A. DC., grupo de especies que se reconoce con facilidad dentro de la sección Tomentosae por tener en común ramitas e inflorescencias variadamente hirsutas, con los pelos del indumento usualmente erectos o suberectos. Prestonia acrensis puede ser fácilmente separada de estas especies por sus flores, con los lóbulos ovados, 4-5 x 2-3 mm (vs. obovados y de 8$16 \times 6-10 \mathrm{~mm}$ ) e inflorescencias racemosas (vs. umbeliformes a subumbeliformes). Caracteres adicionales que distinguen $P$. acrensis de P. ipomaeifolia incluyen hojas con láminas foliares mucho más pequeñas y en relación a $P$. calycina, flores con el tubo más pequeño (9-14 mm vs. 15$21 \mathrm{~mm}$ ) y anteras más pequeñas (4.5-5 mm vs. 5.5-7 mm).

Prestonia acrensis puede ser confundida con la común y ampliamente distribuida $P$. tomentos $a \mathrm{R}$. Br., pero esta última especie tiene la pubescencia de tallos e inflorescencias no hirsutas, sino usualmente tomentosa o tomentulosa, con el indumento corto, ferrugíneo y más o menos adpreso. Otros caracteres diferentes en $P$. acrensis en relación a $P$. tomentosa incluyen inflorescencias racemosas (vs. umbeliformes a subumbeliformes o raramente subracemosas) y lóbulos de la corola más pequeños (4-5 x 2-3 mm vs. 7.5-13 x 5-8 mm).

Paratipos. Brasil. Amazonas: Igarapé de Flores, May 1955 (fl), Chagas s.n. (NY).

\section{AGRADECIMIENTOS}

Quiero agradecer a los curadores de los herbarios INPA y NY por el material enviado en préstamo.

\section{LITERATURA CITADA}

Morales, J. F. 1997. A synopsis of the genus Prestonia (Apocynaceae) section Tomentosae in Mesoamerica. Novon 7(1): 59-66

Sales, M. F. 1993. Estudos taxonômicos do gênero Mandevilla Lindley subgênero Mandevilla (Apocynaceae) no Brasil. Tese do doctorado. Universidade Estadual do Campinas, Sâo Paulo. Brasil.

Woodson, R. E. 1936. Studies in the Apocynaceae. IV. The American genera of Echitoideae. Ann. Missouri Bot. Gard. 23(2): 276-436.

RECEBIDO EM: 31/07/2003

ACEITO EM: 02/04/2004 